\title{
Nonlinear Oscillation and Asymptotic Behavior of Second-order Neutral Dynamic Equations on Time Scales
}

\author{
Daxue Chen \\ College of Science, Hunan Institute of Engineering \\ 88 East Fuxing Road, Xiangtan 411104, Hunan, China \\ E-mail: cdx2003@163.com
}

Received: January 18, 2011

Accepted: February 13, 2011

doi:10.5539/mas.v5n2p179

The research is financed by the Science and Technology Program of Hunan Province of P. R. China (Grant No. 2010FJ6021).

\begin{abstract}
The paper is to study the oscillation and asymptotic behavior of the second-order nonlinear neutral dynamic equation

$$
\left(r(t)\left|y^{\Delta}(t)\right|^{\alpha} \operatorname{sgn} y^{\Delta}(t)\right)^{\Delta}+q(t)|x(t)|^{\beta} \operatorname{sgn} x(t)=0
$$

on an arbitrary time scale $\mathrm{T}$, where $\alpha \geq 1, \beta>0$ are constants, $r, q \in C_{r d}(\mathrm{~T},(0, \infty)), y(t):=$ $x(t)+p(t) x(\tau(t)), \quad p \in C_{r d}(\mathrm{~T},[0,1]), \quad \tau \in C_{r d}(\mathrm{~T}, \mathrm{~T}), \tau(t) \leq t$ for $t \in \mathrm{T}$ and $\lim _{t \rightarrow \infty} \tau(t)=\infty$. By using a generalized Riccati transformation technique, we obtain some sufficient conditions which ensure that every solution of the equation oscillates or converges to zero. Our results improve and extend some existing results in which $p(t) \equiv 0$ and $\alpha, \beta$ are quotients of odd positive integers.
\end{abstract}

Keywords: Nonlinear oscillation, Asymptotic behavior, Neutral dynamic equation, Time scale

\section{Introduction}

In this paper, we consider the oscillation and asymptotic behavior of solutions of the following nonlinear second-order neutral dynamic equation

$$
\left(r(t)\left|y^{\Delta}(t)\right|^{\alpha} \operatorname{sgn} y^{\Delta}(t)\right)^{\Delta}+q(t)|x(t)|^{\beta} \operatorname{sgn} x(t)=0
$$

on an arbitrary time scale $\mathrm{T}$, where

$$
y(t):=x(t)+p(t) x(\tau(t))
$$

and the following conditions are assumed to hold:

$$
\left.\begin{array}{l}
\alpha \geq 1, \beta>0 \text { are constants, } r, q \in C_{r d}(\mathrm{~T},(0, \infty)), \sup \mathrm{T}=\infty, p \in C_{r d}(\mathrm{~T}, \square), \\
0 \leq p(t) \leq 1 \text { for } t \in \mathrm{T}, \tau \in C_{r d}(\mathrm{~T}, \mathrm{~T}), \tau(t) \leq t \text { for } t \in \mathrm{T} \text { and } \lim _{t \rightarrow \infty} \tau(t)=\infty .
\end{array}\right\}
$$

Recall that a solution of (1) is a nontrivial real function $x$ such that

$$
x(t)+p(t) x(\tau(t)) \in C_{r d}^{1}\left[t_{x}, \infty\right) \text { and } r(t)\left|y^{\Delta}(t)\right|^{\alpha} \operatorname{sgn} y^{\Delta}(t) \in C_{r d}^{1}\left[t_{x}, \infty\right)
$$

for a certain $t_{x} \geq t_{0}$ and satisfying (1) for $t \geq t_{x}$. Our attention is restricted to those solutions of (1) which exist on the half-line $\left[t_{x}, \infty\right)$ and satisfy $\sup \left\{|x(t)|: t>t_{*}\right\}>0$ for any $t_{*} \geq t_{x}$. A solution $x$ of (1) is said to be oscillatory if it is neither eventually positive nor eventually negative. Otherwise it is nonoscillatory. Equation (1) is said to be oscillatory if all its solutions are oscillatory.

The concept of dynamic equations on time scales was introduced by Hilger in his PhD thesis (Hilger, 1990) with the motivation of providing a unified approach to continuous and discrete calculus. Thus, the notion of a generalized delta derivative $f^{\Delta}(t)$ was introduced, where the domain of the function $f$ is a so-called "time 
scale" $\mathrm{T}$ (an arbitrary nonempty closed subset of $\square$ ). If the time scale $\mathrm{T}$ is the real numbers $\square$, then the usual derivative is retrieved, that is, $f^{\Delta}(t)=f^{\prime}(t)$. On the other hand, if the time scale $\mathrm{T}$ is taken to be the integers $\square$, then the generalized delta derivative reduces to the usual forward difference, that is, $f^{\Delta}(t)=\Delta f(t)=f(t+1)-f(t)$. Not only can the theory of dynamic equations on time scales unify the theories of differential equations and difference equations, but it is also able to extend these classical cases to cases "in between," e.g., to the so-called $q$-difference equations. For an introduction to time scale calculus and dynamic equations, we refer to the seminal book by Bohner and Peterson (2001). For advances in dynamic equations on time scales, one can see the book by Bohner and Peterson (2003). Throughout the paper it is assumed that the reader is familiar with time scale calculus.

In the last years, a lot of authors have investigated the oscillatory and asymptotic behavior of solutions of different classes of dynamic equations on time scales, and we refer the reader to (Chen \& Liu, 2008; Chen, 2010a; Chen, 2010b; Chen \& Liu, 2010; Grace et al., 2008; Grace et al., 2009; Hassan, 2008; Karpuz, 2009; Saker, 2005; Saker \& O’Regan, 2011; Tripathy, 2009; Xu \& Xu, 2009).

Recently, Saker (2005) established some oscillation criteria for (1) when $p(t) \equiv 0$ and $\alpha=\beta>1$ is an odd positive integer. Hassan (2008) obtained some sufficient conditions for the oscillation of (1) when $p(t) \equiv 0$ and $\alpha=\beta$ is a quotient of odd positive integers. Hassan (2008) improved and extended the results of Saker (2005). Grace et al. $(2008,2009)$ gave some new oscillation results for (1) when $p(t) \equiv 0$ and $\alpha, \beta$ are quotients of odd positive integers.

It is easy to see that the cases considered in (Saker, 2005; Hassan, 2008; Grace et al., 2008; Grace et al., 2009) only are some special cases of (1) and that all the results of (Saker, 2005; Hassan, 2008; Grace et al., 2008; Grace et al., 2009) can not be applied to (1) when $p(t)$ is not identically equal to zero or $\alpha, \beta$ are not equal to quotients of odd positive integers. Accordingly, it is of great interest to study the oscillation and asymptotic behavior of (1) when $p(t)$ is not identically equal to zero and $\alpha, \beta>0$ are constants. In this paper, we will establish some new oscillation criteria for (1) when $0 \leq p(t) \leq 1$ for $t \in \mathrm{T}$ and $\alpha \geq 1, \beta>0$ are constants. We don't restrict $\alpha=\beta$, which was used in (Saker, 2005) and (Hassan, 2008). Our results improve and extend some of those in (Saker, 2005; Hassan, 2008; Grace et al., 2008; Grace et al., 2009).

The following lemma will play an important role in the proof of our main results.

Lemma 1. ((Bohner and Peterson, 2001), p. 32, Theorem 1.87) Let $f: \mathrm{R} \rightarrow \mathrm{R}$ be continuously differentiable and suppose $g: \mathrm{T} \rightarrow \mathrm{R}$ is delta differentiable. Then $f \circ g: \mathrm{T} \rightarrow \mathrm{R}$ is delta differentiable and satisfies

$$
(f \circ g)^{\Delta}(t)=\left\{\int_{0}^{1} f^{\prime}\left(g(t)+h \mu(t) g^{\Delta}(t)\right) d h\right\} g^{\Delta}(t),
$$

where $\mu(t):=\sigma(t)-t$ is the graininess function on $\mathrm{T}$, here $\sigma(t):=\inf \{s \in \mathrm{Tt} s>t\}$ is the forward jump operator on $\mathrm{T}$.

In what follows, for convenience, when we write a functional inequality without specifying its domain of validity we assume that it holds for all sufficiently large $t$.

\section{Main results}

In this section, we will present and prove our main results. We will consider both the case when

$$
\int_{t_{0}}^{\infty} r^{-1 / \alpha}(t) \Delta t=\infty
$$

holds and the case when

$$
\int_{t_{0}}^{\infty} r^{-1 / \alpha}(t) \Delta t<\infty
$$

holds.

Theorem 1. Suppose that (3) and (4) hold. Furthermore, assume that there exists a positive delta differentiable function $\varphi$ such that for all $t_{4}>t_{3} \geq t_{0}$, 


$$
\limsup _{t \rightarrow \infty} \int_{t_{4}}^{t}\left\{q(s)[1-p(s)]^{\beta} \varphi(s)-\frac{r^{1 / \alpha}(s)\left(\varphi^{\Delta}(s)\right)^{2}\left(\Phi^{\sigma}(s)\right)^{1-\alpha}}{4 \beta \varphi(s) \psi(s)}\right\} \Delta s=\infty
$$

where $\Phi(t):=\int_{t_{3}}^{t} r^{-1 / \alpha}(s) \Delta s$ and $\psi(t):= \begin{cases}c_{1}, & \text { if } \alpha<\beta, \\ 1, & \text { if } \alpha=\beta, \text { here } c_{1} \text { and } c_{2} \text { are any } \\ c_{2}\left(\Phi^{\sigma}(t)\right)^{\beta-\alpha}, & \text { if } \alpha>\beta,\end{cases}$ positive constants, $\sigma$ is the forward jump operator on $\mathrm{T}$ and $\Phi^{\sigma}:=\Phi \circ \sigma$. Then (1) is oscillatory.

Proof. Assume that $x$ is a nonoscillatory solution of (1). Without loss of generality, we may assume that $x$ is an eventually positive solution of (1). Then it follows from (2) and (3) that there exists $t_{1} \geq t_{0}$ such that

$$
y(t) \geq x(t)>0 \text { for } t \in\left[t_{1}, \infty\right) .
$$

Thus, from (1) we get

$$
\left(r(t)\left|y^{\Delta}(t)\right|^{\alpha} \operatorname{sgn} y^{\Delta}(t)\right)^{\Delta}=-q(t) x^{\beta}(t)<0 \text { for } t \in\left[t_{1}, \infty\right) .
$$

Therefore, we conclude that $r(t)\left|y^{\Delta}(t)\right|^{\alpha} \operatorname{sgn} y^{\Delta}(t)$ is strictly decreasing on $\left[t_{1}, \infty\right)$ and eventually of one sign. Hence, $y^{\Delta}(t)$ is eventually of one sign. We now claim

$$
y^{\Delta}(t)>0 \text { for } t \in\left[t_{1}, \infty\right) .
$$

If (9) doesn't hold, then $y^{\Delta}(t)$ is eventually negative and there exists $t_{2} \geq t_{1}$ such that $y^{\Delta}\left(t_{2}\right)<0$. Since $r(t)\left|y^{\Delta}(t)\right|^{\alpha} \operatorname{sgn} y^{\Delta}(t)$ is strictly decreasing on $\left[t_{1}, \infty\right)$, we obtain

$$
r(t)\left|y^{\Delta}(t)\right|^{\alpha} \operatorname{sgn} y^{\Delta}(t) \leq r\left(t_{2}\right)\left|y^{\Delta}\left(t_{2}\right)\right|^{\alpha} \operatorname{sgn} y^{\Delta}\left(t_{2}\right):=c_{0}<0 \text { for } t \in\left[t_{2}, \infty\right) .
$$

Then we get $y^{\Delta}(t) \leq-\left(-c_{0}\right)^{1 / \alpha} r^{-1 / \alpha}(t)$ for $t \in\left[t_{2}, \infty\right)$. Integrating both sides of the last inequality from $t_{2}$ to $t$, we have $y(t)-y\left(t_{2}\right) \leq-\left(-c_{0}\right)^{1 / \alpha} \int_{t_{2}}^{t} r^{-1 / \alpha}(s) \Delta s$ for $t \in\left[t_{2}, \infty\right)$. Letting $t \rightarrow \infty$ and using (4), we conclude $\lim _{t \rightarrow \infty} y(t)=-\infty$. This contradicts (7). Thus, we get that (9) holds. From (2) and (7), we have $y(\tau(t)) \geq x(\tau(t))$ and

$$
x(t)=y(t)-p(t) x(\tau(t)) \geq y(t)-p(t) y(\tau(t)) .
$$

Since $\tau(t) \leq t$, from (9) we get $y(\tau(t)) \leq y(t)$. Hence, from (11) we obtain

$$
x(t) \geq y(t)-p(t) y(t)=[1-p(t)] y(t) .
$$

From (8), (9) and (12), there exists $t_{3} \in\left[t_{2}, \infty\right)$ such that

$$
\left[r(t)\left(y^{\Delta}(t)\right)^{\alpha}\right]^{\Delta} \leq-q(t)[1-p(t)]^{\beta} y^{\beta}(t) \leq 0 \text { for } t \in\left[t_{3}, \infty\right) .
$$

Define the function $w$ by

$$
w(t):=r(t)\left(y^{\Delta}(t)\right)^{\alpha} \frac{\varphi(t)}{y^{\beta}(t)} \text { for } t \in\left[t_{3}, \infty\right) .
$$

Then we have $w(t)>0$ for $t \in\left[t_{3}, \infty\right)$. By the formulas

$$
(f g)^{\Delta}=f^{\Delta} g+f^{\sigma} g^{\Delta} \text { and }(f / g)^{\Delta}=f^{\Delta} / g^{\sigma}-f g^{\Delta} /\left(g g^{\sigma}\right)
$$

for the delta derivatives of the product $f g$ and the quotient $f / g$ of differentiable functions $f$ and $g$, where $\sigma$ is the forward jump operator on $\mathrm{T}, f^{\sigma}:=f \circ \sigma$ and $g^{\sigma}:=g \circ \sigma$, we get for $t \in\left[t_{3}, \infty\right)$

$$
w^{\Delta}=\left(r\left(y^{\Delta}\right)^{\alpha}\right)^{\Delta} \frac{\varphi}{y^{\beta}}+\left(r\left(y^{\Delta}\right)^{\alpha}\right)^{\sigma}\left(\frac{\varphi}{y^{\beta}}\right)^{\Delta}=\left(r\left(y^{\Delta}\right)^{\alpha}\right)^{\Delta} \frac{\varphi}{y^{\beta}}+\left(r\left(y^{\Delta}\right)^{\alpha}\right)^{\sigma}\left(\frac{\varphi^{\Delta}}{\left(y^{\beta}\right)^{\sigma}}-\frac{\varphi\left(y^{\beta}\right)^{\Delta}}{y^{\beta}\left(y^{\beta}\right)^{\sigma}}\right) \text {. }
$$

Hence, from (13), (14) and the last equality we obtain

$$
w^{\Delta} \leq-q(1-p)^{\beta} \varphi+\left(r\left(y^{\Delta}\right)^{\alpha}\right)^{\sigma}\left(\frac{\varphi^{\Delta}}{\left(y^{\beta}\right)^{\sigma}}-\frac{\varphi\left(x^{\beta}\right)^{\Delta}}{y^{\beta}\left(y^{\beta}\right)^{\sigma}}\right)
$$




$$
\leq-q(1-p)^{\beta} \varphi+\frac{w^{\sigma}}{\varphi^{\sigma}} \varphi^{\Delta}-\varphi \frac{w^{\sigma}}{\varphi^{\sigma}} \frac{\left(y^{\beta}\right)^{\Delta}}{y^{\beta}} \text { on }\left[t_{3}, \infty\right) .
$$

Since $0<y(t) \leq y^{\sigma}(t)$ for $t \in\left[t_{3}, \infty\right)$, by the formula $y^{\sigma}(t)=y(t)+\mu(t) y^{\Delta}(t)$ and by Lemma 1 , we obtain for $t \in\left[t_{3}, \infty\right)$

$$
\begin{aligned}
\left(y^{\beta}(t)\right)^{\Delta} & =\beta y^{\Delta}(t) \int_{0}^{1}\left[y(t)+h \mu(t) y^{\Delta}(t)\right]^{\beta-1} d h=\beta y^{\Delta}(t) \int_{0}^{1}\left[(1-h) y(t)+h y^{\sigma}(t)\right]^{\beta-1} d h \\
& \geq\left\{\begin{array}{ll}
\beta y^{\Delta}(t) \int_{0}^{1}\left(y^{\sigma}(t)\right)^{\beta-1} d h, & \text { if } 0<\beta \leq 1, \\
\beta y^{\Delta}(t) \int_{0}^{1} y^{\beta-1}(t) d h, & \text { if } \beta>1
\end{array}= \begin{cases}\beta\left(y^{\sigma}(t)\right)^{\beta-1} y^{\Delta}(t), & \text { if } 0<\beta \leq 1, \\
\beta y^{\beta-1}(t) y^{\Delta}(t), & \text { if } \beta>1 .\end{cases} \right.
\end{aligned}
$$

Therefore, for $t \in\left[t_{3}, \infty\right)$ we conclude

$$
\frac{\left(y^{\beta}(t)\right)^{\Delta}}{y^{\beta}(t)} \geq \begin{cases}\beta\left(y^{\sigma}(t)\right)^{\beta-1} y^{\Delta}(t) / y^{\beta}(t), & \text { if } 0<\beta \leq 1, \\ \beta y^{\Delta}(t) / y(t), & \text { if } \beta>1 .\end{cases}
$$

Since $0<y(t) \leq y^{\sigma}(t)$ for $t \in\left[t_{3}, \infty\right)$, from (16) we obtain

$$
\left(y^{\beta}(t)\right)^{\Delta} / y^{\beta}(t) \geq \beta y^{\Delta}(t) / y^{\sigma}(t) \text { for } t \in\left[t_{3}, \infty\right) \text { and for all } \beta>0 .
$$

Hence, from (15) and (17) we find

$$
w^{\Delta} \leq-q(1-p)^{\beta} \varphi+\frac{w^{\sigma}}{\varphi^{\sigma}} \varphi^{\Delta}-\beta \varphi \frac{w^{\sigma}}{\varphi^{\sigma}} \frac{y^{\Delta}}{y^{\sigma}} \text { on }\left[t_{3}, \infty\right) .
$$

From (14) we get $r^{1 / \alpha} y^{\Delta}=\left(w y^{\beta} / \varphi\right)^{1 / \alpha}$ on $\left[t_{3}, \infty\right)$. Since $r^{1 / \alpha}(t) y^{\Delta}(t)$ is strictly decreasing and $t \leq \sigma(t)$ on $\left[t_{1}, \infty\right)$, we have $r^{1 / \alpha} y^{\Delta} \geq\left(r^{1 / \alpha} y^{\Delta}\right)^{\sigma}=\left(w^{\sigma}\left(y^{\sigma}\right)^{\beta} / \varphi^{\sigma}\right)^{1 / \alpha}$ on $\left[t_{3}, \infty\right)$. Thus, from (18) we obtain

$$
\begin{aligned}
& w^{\Delta} \leq-q(1-p)^{\beta} \varphi+\frac{w^{\sigma}}{\varphi^{\sigma}} \varphi^{\Delta}-\frac{\beta \varphi\left(w^{\sigma}\right)^{(1+\alpha) / \alpha}}{r^{1 / \alpha}\left(\varphi^{\sigma}\right)^{(1+\alpha) / \alpha}}\left(y^{\sigma}\right)^{(\beta-\alpha) / \alpha} \\
& =-q(1-p)^{\beta} \varphi+\frac{w^{\sigma}}{\varphi^{\sigma}} \varphi^{\Delta}-\frac{\beta \varphi\left(w^{\sigma}\right)^{2}\left(w^{\sigma}\right)^{(1-\alpha) / \alpha}}{r^{1 / \alpha}\left(\varphi^{\sigma}\right)^{(1+\alpha) / \alpha}}\left(y^{\sigma}\right)^{\frac{\beta-\alpha}{\alpha}} \text { for } t \in\left[t_{3}, \infty\right) .
\end{aligned}
$$

It follows from (14) that $w^{\sigma}=r^{\sigma}\left(\left(y^{\Delta}\right)^{\sigma}\right)^{\alpha} \varphi^{\sigma} /\left(y^{\sigma}\right)^{\beta}$ on $\left[t_{3}, \infty\right)$. Therefore, we get

$$
\begin{aligned}
\left(y^{\sigma}\right)^{(\beta-\alpha) / \alpha}\left(w^{\sigma}\right)^{(1-\alpha) / \alpha} & =\left(y^{\sigma}\right)^{(\beta-\alpha) / \alpha}\left[r^{\sigma}\left(\left(y^{\Delta}\right)^{\sigma}\right)^{\alpha} \varphi^{\sigma} /\left(y^{\sigma}\right)^{\beta}\right]^{(1-\alpha) / \alpha} \\
& =\left(y^{\sigma}\right)^{\beta-1}\left(r^{\sigma} \varphi^{\sigma}\right)^{(1-\alpha) / \alpha}\left(\left(y^{\Delta}\right)^{\sigma}\right)^{1-\alpha} \\
& =\left(r^{\sigma} \varphi^{\sigma}\right)^{(1-\alpha) / \alpha}\left(\left(y / y^{\Delta}\right)^{\sigma}\right)^{\alpha-1}\left(y^{\sigma}\right)^{\beta-\alpha} \text { on }\left[t_{3}, \infty\right) .
\end{aligned}
$$

Since $r^{1 / \alpha}(t) y^{\Delta}(t)$ is strictly decreasing on $\left[t_{3}, \infty\right)$, we obtain for $t \in\left[t_{3}, \infty\right)$

$$
\begin{aligned}
y(t)=y\left(t_{3}\right)+\int_{t_{3}}^{t} y^{\Delta}(s) \Delta s & =y\left(t_{3}\right)+\int_{t_{3}}^{t} r^{-1 / \alpha}(s)\left[r^{1 / \alpha}(s) y^{\Delta}(s)\right] \Delta s \\
& \geq r^{1 / \alpha}(t) y^{\Delta}(t) \int_{t_{3}}^{t} r^{-1 / \alpha}(s) \Delta s .
\end{aligned}
$$

Thus, we get $\frac{y(t)}{y^{\Delta}(t)} \geq r^{1 / \alpha}(t) \int_{t_{3}}^{t} r^{-1 / \alpha}(s) \Delta s:=r^{1 / \alpha}(t) \Phi(t)$ for $t \in\left[t_{3}, \infty\right)$, where $\Phi$ is defined as in Theorem 1. Since $\alpha \geq 1$, we have $\left[\left(y / y^{\Delta}\right)^{\sigma}\right]^{\alpha-1} \geq\left(r^{\sigma}\right)^{(\alpha-1) / \alpha}\left(\Phi^{\sigma}\right)^{\alpha-1}$ on $\left(t_{3}, \infty\right)$. Hence, from (20) 
we conclude

$$
\left(y^{\sigma}\right)^{(\beta-\alpha) / \alpha}\left(w^{\sigma}\right)^{(1-\alpha) / \alpha} \geq\left(\varphi^{\sigma}\right)^{(1-\alpha) / \alpha}\left(\Phi^{\sigma}\right)^{\alpha-1}\left(y^{\sigma}\right)^{\beta-\alpha} \text { on }\left(t_{3}, \infty\right) .
$$

Then from (19) we obtain

$$
w^{\Delta} \leq-q(1-p)^{\beta} \varphi+\frac{w^{\sigma}}{\varphi^{\sigma}} \varphi^{\Delta}-\frac{\beta \varphi\left(w^{\sigma}\right)^{2}}{r^{1 / \alpha}\left(\varphi^{\sigma}\right)^{2}}\left(\Phi^{\sigma}\right)^{\alpha-1}\left(y^{\sigma}\right)^{\beta-\alpha} \text { on }\left(t_{3}, \infty\right) .
$$

Next, we consider the following three cases:

Case (i). Let $\alpha<\beta$. Since $y^{\sigma}(t) \geq y(t) \geq y\left(t_{3}\right)>0$ for $t \in\left[t_{3}, \infty\right)$, we have

$$
\left(y^{\sigma}\right)^{\beta-\alpha}(t) \geq\left(y\left(t_{3}\right)\right)^{\beta-\alpha}:=c_{1} \text { for } t \in\left[t_{3}, \infty\right) .
$$

Case (ii). Let $\alpha=\beta$. Then we get

$$
\left(y^{\sigma}\right)^{\beta-\alpha}(t)=1 \text { for } t \in\left[t_{3}, \infty\right) .
$$

Case (iii). Let $\alpha>\beta$. Since $r(t)\left(y^{\Delta}(t)\right)^{\alpha}$ is strictly decreasing on $\left[t_{3}, \infty\right)$, for $t \in\left[t_{3}, \infty\right)$ we have $r(t)\left(y^{\Delta}(t)\right)^{\alpha} \leq r\left(t_{3}\right)\left(y^{\Delta}\left(t_{3}\right)\right)^{\alpha}:=b$. Hence, we obtain $y^{\Delta}(t) \leq b^{1 / \alpha} r^{-1 / \alpha}(t)$ for $t \in\left[t_{3}, \infty\right)$. Integrating both sides of the last inequality from $t_{3}$ to $t$, we have

$$
y(t) \leq y\left(t_{3}\right)+b^{1 / \alpha} \int_{t_{3}}^{t} r^{-1 / \alpha}(s) \Delta s \text { for } t \in\left[t_{3}, \infty\right) .
$$

Therefore, there exist a constant $b_{1}>0$ and $t_{4}>t_{3}$ such that $y(t) \leq b_{1} \int_{t_{3}}^{t} r^{-1 / \alpha}(s) \Delta s:=b_{1} \Phi(t)$ for $t \in\left[t_{4}, \infty\right)$. Hence, we get

$$
\left(y^{\sigma}(t)\right)^{\beta-\alpha} \geq b_{1}^{\beta-\alpha}\left(\Phi^{\sigma}(t)\right)^{\beta-\alpha}:=c_{2}\left(\Phi^{\sigma}(t)\right)^{\beta-\alpha} \text { for } t \in\left[t_{4}, \infty\right) .
$$

Thus, for all $\alpha \geq 1, \beta>0$ and for $t \in\left[t_{4}, \infty\right)$, from (21)-(24) it follows that

$$
\begin{gathered}
w^{\Delta} \leq-q(1-p)^{\beta} \varphi+\frac{w^{\sigma}}{\varphi^{\sigma}} \varphi^{\Delta}-\frac{\beta \varphi\left(w^{\sigma}\right)^{2}}{r^{1 / \alpha}\left(\varphi^{\sigma}\right)^{2}}\left(\Phi^{\sigma}\right)^{\alpha-1} \psi \\
=-q(1-p)^{\beta} \varphi+\frac{r^{1 / \alpha}\left(\varphi^{\Delta}\right)^{2}\left(\Phi^{\sigma}\right)^{1-\alpha}}{4 \beta \varphi \psi}-\left[\sqrt{\frac{\beta \varphi\left(w^{\sigma}\right)^{2}\left(\Phi^{\sigma}\right)^{\alpha-1} \psi}{r^{1 / \alpha}\left(\varphi^{\sigma}\right)^{2}}}-\varphi^{\Delta} \sqrt{\frac{r^{1 / \alpha}\left(\Phi^{\sigma}\right)^{1-\alpha}}{4 \beta \varphi \psi}}\right]^{2} \\
\leq-q(1-p)^{\beta} \varphi+\frac{r^{1 / \alpha}\left(\varphi^{\Delta}\right)^{2}\left(\Phi^{\sigma}\right)^{1-\alpha}}{4 \beta \varphi \psi} \text { on }\left[t_{4}, \infty\right),
\end{gathered}
$$

where $\psi$ is defined as in Theorem 1. Integrating both sides of the last inequality from $t_{4}$ to $t$, we obtain for $t \in\left[t_{4}, \infty\right)$

$$
\int_{t_{4}}^{t}\left\{q(s)[1-p(s)]^{\beta} \varphi(s)-\frac{r^{1 / \alpha}(s)\left(\varphi^{\Delta}(s)\right)^{2}\left(\Phi^{\sigma}(s)\right)^{1-\alpha}}{4 \beta \varphi(s) \psi(s)}\right\} \Delta s \leq w\left(t_{4}\right)-w(t)<w\left(t_{4}\right) .
$$

Therefore, we have

$$
\limsup _{t \rightarrow \infty} \int_{t_{4}}^{t}\left\{q(s)[1-p(s)]^{\beta} \varphi(s)-\frac{r^{1 / \alpha}(s)\left(\varphi^{\Delta}(s)\right)^{2}\left(\Phi^{\sigma}(s)\right)^{1-\alpha}}{4 \beta \varphi(s) \psi(s)}\right\} \Delta s \leq w\left(t_{4}\right)<\infty,
$$

which implies a contradiction to (6). The proof is complete.

Theorem 2. Suppose that (3) and (5) hold. Furthermore, assume that there exists a positive delta differentiable function $\varphi$ such that (6) holds and that there exists a constant $\varepsilon>0$ such that the following conditions hold: 


$$
1-(1+\varepsilon) p(t) \geq 0 \text { for } t \in \mathrm{T}
$$

and

$$
\int_{T}^{\infty}\left\{r^{-1}(v) \int_{T}^{v} q(u)[1-(1+\varepsilon) p(u)]^{\beta} \Delta u\right\}^{1 / \alpha} \Delta v=\infty \text { for all } T \geq t_{0} .
$$

Then every solution of (1) is oscillatory or converges to zero as $t \rightarrow \infty$.

Proof. Assume that $x$ is a nonoscillatory solution of (1). Without loss of generality, we may assume that $x$ is an eventually positive solution of (1). Then there exists $t_{1} \geq t_{0}$ such that (7) and (8) hold. Therefore, we see that $r(t)\left|y^{\Delta}(t)\right|^{\alpha} \operatorname{sgn} y^{\Delta}(t)$ is strictly decreasing on $\left[t_{1}, \infty\right)$ and eventually of one sign. Hence, $y^{\Delta}(t)$ is eventually of one sign, i.e., there are the following two cases for the sign of $y^{\Delta}(t)$ : Case (i). $y^{\Delta}(t)$ is eventually positive; Case (ii). $y^{\Delta}(t)$ is eventually negative. The proof of Case (i) is similar to that of Theorem 1 and hence is omitted.

Next, we only consider Case (ii). In this case, there exists $t_{2} \geq t_{1}$ such that $y^{\Delta}(t)<0$ for $t \in\left[t_{2}, \infty\right)$. In view of (7), we have $\lim _{t \rightarrow \infty} y(t):=L \geq 0$ and $y(t) \geq L$. We claim $L=0$. If not, then we have $L>0$ and we will show that this leads to a contradiction to (27). By the properties of limit, for $\varepsilon>0$ we have

$$
L \leq y(t)<(1+\varepsilon) L \text {. }
$$

Thus, we obtain

$$
L \leq y(\tau(t))<(1+\varepsilon) L .
$$

From (7), we have $y(\tau(t)) \geq x(\tau(t))$. Therefore, from (2) we get

$$
x(t)=y(t)-p(t) x(\tau(t)) \geq y(t)-p(t) y(\tau(t)) .
$$

It follows from (26) and (28)-(30) that

$$
x(t) \geq y(t)-p(t) y(\tau(t)) \geq L[1-(1+\varepsilon) p(t)] \geq 0 .
$$

From (8) and (31), there exists $t_{3} \in\left[t_{2}, \infty\right)$ such that

$$
\left[r(t)\left(-y^{\Delta}(t)\right)^{\alpha}\right]^{\Delta}=q(t) x^{\beta}(t) \geq L^{\beta} q(t)[1-(1+\varepsilon) p(t)]^{\beta} \text { for } t \in\left[t_{3}, \infty\right) .
$$

Integrating both sides of the last inequality from $t_{3}$ to $t$, we have for $t \in\left[t_{3}, \infty\right)$

$$
\begin{aligned}
r(t)\left(-y^{\Delta}(t)\right)^{\alpha} & \geq r\left(t_{3}\right)\left(-y^{\Delta}\left(t_{3}\right)\right)^{\alpha}+L^{\beta} \int_{t_{3}}^{t} q(u)[1-(1+\varepsilon) p(u)]^{\beta} \Delta u \\
& \geq L^{\beta} \int_{t_{3}}^{t} q(u)[1-(1+\varepsilon) p(u)]^{\beta} \Delta u .
\end{aligned}
$$

Hence, we obtain $y^{\Delta}(t) \leq-L^{\beta / \alpha}\left\{r^{-1}(t) \int_{t_{3}}^{t} q(u)[1-(1+\varepsilon) p(u)]^{\beta} \Delta u\right\}^{1 / \alpha}$ for $t \in\left[t_{3}, \infty\right)$. Integrating both sides of the last inequality from $t_{3}$ to $t$, we have

$$
y(t) \leq y\left(t_{3}\right)-L^{\beta / \alpha} \int_{t_{3}}^{t}\left\{r^{-1}(v) \int_{t_{3}}^{v} q(u)[1-(1+\varepsilon) p(u)]^{\beta} \Delta u\right\}^{1 / \alpha} \Delta v \text { for } t \in\left[t_{3}, \infty\right) .
$$

Therefore, we conclude for $t \in\left[t_{3}, \infty\right)$

$$
\int_{t_{3}}^{t}\left\{r^{-1}(v) \int_{t_{3}}^{v} q(u)[1-(1+\varepsilon) p(u)]^{\beta} \Delta u\right\}^{1 / \alpha} \Delta v \leq\left[y\left(t_{3}\right)-y(t)\right] / L^{\beta / \alpha}<y\left(t_{3}\right) / L^{\beta / \alpha} .
$$

Letting $t \rightarrow \infty$, we obtain $\int_{t_{3}}^{\infty}\left\{r^{-1}(v) \int_{t_{3}}^{v} q(u)[1-(1+\varepsilon) p(u)]^{\beta} \Delta u\right\}^{1 / \alpha} \Delta v \leq y\left(t_{3}\right) / L^{\beta / \alpha}<\infty$, which yields a contradiction to (27). Therefore we have $L=0$, i.e., $\lim _{t \rightarrow \infty} y(t)=0$. In view of (7), we obtain $\lim _{t \rightarrow \infty} x(t)=0$. The proof is complete.

In order to present our next theorems, we now introduce the class of functions $\mathfrak{R}$.

Let $\quad \mathrm{D}:=\left\{(t, s) \in \mathrm{T} \times \mathrm{T}: t \geq s \geq t_{0}\right\} \quad$ and $\quad \mathrm{D}_{0}:=\left\{(t, s) \in \mathrm{T} \times \mathrm{T}: t>s \geq t_{0}\right\} \quad$. The function 
$H \in C_{r d}(\mathrm{D}, \mathrm{R})$ is said to belong to the class $\mathfrak{R}$ if $H(t, t) \geq 0$ for $t \geq t_{0}, H(t, s)>0$ for $(t, s) \in \mathrm{D}_{00}$, and $H$ has a rd-continuous delta partial derivative $H^{\Delta_{s}}(t, s)$ on $\mathrm{D}_{0}$ with respect to the second variable.

Theorem 3. Assume that (3) and (4) hold and that $H$ belongs to $\mathfrak{R}$. Furthermore, assume that there exist a positive delta differentiable function $\varphi \in C_{r d}(\mathrm{~T}, \square)$ and a function $h \in C_{r d}(\mathrm{D}, \square)$ such that

$$
\varphi^{\Delta}(s) H(t, s) / \varphi^{\sigma}(s)+H^{\Delta_{s}}(t, s)=h(t, s) \sqrt{H(t, s)} / \varphi^{\sigma}(s) \text { for } t>s \geq t_{0}
$$

and

$$
\limsup _{t \rightarrow \infty} \frac{1}{H(t, T)} \int_{T}^{t-1}\left\{q(s)[1-p(s)]^{\beta} \varphi(s) H(t, s)-\frac{r^{1 / \alpha}(s)\left(\Phi^{\sigma}(s)\right)^{1-\alpha} h^{2}(t, s)}{4 \beta \varphi(s) \psi(s)}\right\} \Delta s=\infty
$$

for all $T>t_{3} \geq t_{0}$, where $\Phi$ and $\psi$ are defined as in Theorem 1 and $\sigma$ is the forward jump operator on $\mathrm{T}$. Then (1) is oscillatory.

Proof. Suppose that $x$ is a nonoscillatory solution of (1). Without loss of generality, we may assume that $x$ is an eventually positive solution of (1). Proceeding as in the proof of Theorem 1, we obtain (25). Multiplying (25) by $H(t, s)$ and then integrating the resulting inequality from $t_{4}$ to $t-1$, we conclude for $t \in\left[t_{4}+1, \infty\right)$

$$
\begin{array}{r}
\int_{t_{4}}^{t-1} q(s)[1-p(s)]^{\beta} \varphi(s) H(t, s) \Delta s \leq-\int_{t_{4}}^{t-1} H(t, s) w^{\Delta}(s) \Delta s+\int_{t_{4}}^{t-1} \frac{w^{\sigma}(s)}{\varphi^{\sigma}(s)} \varphi^{\Delta}(s) H(t, s) \Delta s \\
-\int_{t_{4}}^{t-1} \frac{\beta \varphi(s)\left(w^{\sigma}(s)\right)^{2}}{r^{1 / \alpha}(s)\left(\varphi^{\sigma}(s)\right)^{2}}\left(\Phi^{\sigma}(s)\right)^{\alpha-1} \psi(s) H(t, s) \Delta s .
\end{array}
$$

Using the integration by parts formula

$$
\int_{a}^{b} f(s) g^{\Delta}(s) \Delta s=f(b) g(b)-f(a) g(a)-\int_{a}^{b} f^{\Delta}(s) g^{\sigma}(s) \Delta s,
$$

we have

$$
\begin{aligned}
&-\int_{t_{4}}^{t-1} H(t, s) w^{\Delta}(s) \Delta s=- H(t, t-1) w(t-1)+H\left(t, t_{4}\right) w\left(t_{4}\right)+\int_{t_{4}}^{t-1} H^{\Delta_{s}}(t, s) w^{\sigma}(s) \Delta s \\
& \leq H\left(t, t_{4}\right) w\left(t_{4}\right)+\int_{t_{4}}^{t-1} H^{\Delta_{s}}(t, s) w^{\sigma}(s) \Delta s \text { for } t \in\left[t_{4}+1, \infty\right) .
\end{aligned}
$$

Substituting (36) in (35), we obtain for $t \in\left[t_{4}+1, \infty\right)$

$$
\begin{array}{r}
\int_{t_{4}}^{t-1} q(s)[1-p(s)]^{\beta} \varphi(s) H(t, s) \Delta s \leq H\left(t, t_{4}\right) w\left(t_{4}\right)+\int_{t_{4}}^{t-1}\left[\frac{\varphi^{\Delta}(s)}{\varphi^{\sigma}(s)} H(t, s)+H^{\Delta_{s}}(t, s)\right] w^{\sigma}(s) \Delta s \\
-\int_{t_{4}}^{t-1} \frac{\beta \varphi(s)\left(w^{\sigma}(s)\right)^{2}}{r^{1 / \alpha}(s)\left(\varphi^{\sigma}(s)\right)^{2}}\left(\Phi^{\sigma}(s)\right)^{\alpha-1} \psi(s) H(t, s) \Delta s .
\end{array}
$$

Using (33) in (37), we get

$$
\begin{aligned}
& \int_{t_{4}}^{t-1} q(s)[1-p(s)]^{\beta} \varphi(s) H(t, s) \Delta s \\
& \leq H\left(t, t_{4}\right) w\left(t_{4}\right)+\int_{t_{4}}^{t-1} \frac{h(t, s)}{\varphi^{\sigma}(s)} \sqrt{H(t, s)} w^{\sigma}(s) \Delta s-\int_{t_{4}}^{t-1} \frac{\beta \varphi(s)\left(w^{\sigma}(s)\right)^{2}}{r^{1 / \alpha}(s)\left(\varphi^{\sigma}(s)\right)^{2}}\left(\Phi^{\sigma}(s)\right)^{\alpha-1} \psi(s) H(t, s) \Delta s \\
& =H\left(t, t_{4}\right) w\left(t_{4}\right)+\int_{t_{4}}^{t-1} \frac{r^{1 / \alpha}(s)\left(\Phi^{\sigma}(s)\right)^{1-\alpha} h^{2}(t, s)}{4 \beta \varphi(s) \psi(s)} \Delta s
\end{aligned}
$$




$$
\begin{aligned}
& \quad-\int_{t_{4}}^{t-1}\left[h(t, s) \sqrt{\frac{r^{1 / \alpha}(s)\left(\Phi^{\sigma}(s)\right)^{1-\alpha}}{4 \beta \varphi(s) \psi(s)}}-\sqrt{\frac{\beta \varphi(s)\left(w^{\sigma}(s)\right)^{2}}{r^{1 / \alpha}(s)\left(\varphi^{\sigma}(s)\right)^{2}}\left(\Phi^{\sigma}(s)\right)^{\alpha-1} \psi(s) H(t, s)}\right]^{2} \Delta s \\
& \leq H\left(t, t_{4}\right) w\left(t_{4}\right)+\int_{t_{4}}^{t-1} \frac{r^{1 / \alpha}(s)\left(\Phi^{\sigma}(s)\right)^{1-\alpha} h^{2}(t, s)}{4 \beta \varphi(s) \psi(s)} \Delta s \text { for } t \in\left[t_{4}+1, \infty\right) .
\end{aligned}
$$

Therefore, we have for $t \in\left[t_{4}+1, \infty\right)$

$$
\frac{1}{H\left(t, t_{4}\right)} \int_{t_{4}}^{t-1}\left\{q(s)[1-p(s)]^{\beta} \varphi(s) H(t, s)-\frac{r^{1 / \alpha}(s)\left(\Phi^{\sigma}(s)\right)^{1-\alpha} h^{2}(t, s)}{4 \beta \varphi(s) \psi(s)}\right\} \Delta s \leq w\left(t_{4}\right)
$$

and

$$
\limsup _{t \rightarrow \infty} \frac{1}{H\left(t, t_{4}\right)} \int_{t_{4}}^{t-1}\left\{q(s)[1-p(s)]^{\beta} \varphi(s) H(t, s)-\frac{r^{1 / \alpha}(s)\left(\Phi^{\sigma}(s)\right)^{1-\alpha} h^{2}(t, s)}{4 \beta \varphi(s) \psi(s)}\right\} \Delta s \leq w\left(t_{4}\right)<\infty .
$$

This implies a contradiction to (34). The proof is complete.

Theorem 4. Assume that (3) and (5) hold and that $H$ belongs to $\mathfrak{R}$. Furthermore, assume that there exist a positive delta differentiable function $\varphi \in C_{r d}(\mathrm{~T}, \square)$, a constant $\varepsilon>0$ and a function $h \in C_{r d}(\mathrm{D}, \square)$ such that (26), (27), (33) and (34) hold. Then every solution of (1) is oscillatory or converges to zero as $t \rightarrow \infty$.

Proof. Suppose that $x$ is a nonoscillatory solution of (1). Without loss of generality, we may assume that $x$ is an eventually positive solution of (1). Proceeding as in the proof of Theorem 1, we get (7) and (8). Thus, $r(t)\left|y^{\Delta}(t)\right|^{\alpha} \operatorname{sgn} y^{\Delta}(t)$ is strictly decreasing on $\left[t_{1}, \infty\right)$ and eventually of one sign. Hence, $y^{\Delta}(t)$ is eventually of one sign, i.e., $y^{\Delta}(t)$ is either eventually positive or eventually negative. The proof of the case when $y^{\Delta}(t)$ is eventually positive is similar to that of Theorem 3 and hence is omitted. The proof of the case when $y^{\Delta}(t)$ is eventually negative is similar to that of Case (ii) in the proof of Theorem 2 and thus is omitted. The proof is complete.

\section{Discussion}

The results of this paper are of higher degree of generality. These results can be extended to more general second-order neutral dynamic equation of the form

$$
\left(r(t)\left|y^{\Delta}(t)\right|^{\alpha} \operatorname{sgn} y^{\Delta}(t)\right)^{\Delta}+f(t, x(t))=0,
$$

where $y(t)$ is defined as in (2), $f \in C(\mathrm{~T} \times \square, \square)$ and there exists a positive rd-continuous function $q$ such that

$$
x f(t, x)>0 \text { and }|f(t, x)| \geq q(t)|x|^{\beta} \text { for } x \neq 0 \text { and } t \in\left[t_{0}, \infty\right) .
$$

Our results are also extendable to the second-order neutral delay dynamic equation

$$
\left(r(t)\left|y^{\Delta}(t)\right|^{\alpha} \operatorname{sgn} y^{\Delta}(t)\right)^{\Delta}+f(t, x[\delta(t)])=0,
$$

where $y(t)$ is defined as in (2), $f$ satisfies (38), $\delta: \mathrm{T} \rightarrow \mathrm{T}$ is a rd-continuous function and satisfies $\delta(t) \leq t$ for $t \in \mathrm{T}$ and $\lim _{t \rightarrow \infty} \delta(t)=\infty$. The details are left to the interested reader.

\section{Acknowledgment}

The author is very grateful to the referees for their valuable comments and suggestions, which helped to improve the quality of this paper.

\section{References}

Bohner, M. \& Peterson, A. (2001). Dynamic Equations on Time Scales: An Introduction with Applications. Boston: Birkhäuser.

Bohner, M. \& Peterson, A. (2003). Advances in Dynamic Equations on Time Scales. Boston: Birkhäuser. 
Chen, D. -X. \& Liu, J. -C. (2008). Asymptotic behavior and oscillation of solutions of third-order nonlinear neutral delay dynamic equations on time scales. Can. Appl. Math. Q., 16, 19-43.

Chen, D. -X. (2010a). Oscillation and asymptotic behavior for $n$ th-order nonlinear neutral delay dynamic equations on time scales. Acta Appl. Math., 109, 703-719.

Chen, D. -X. (2010b). Oscillation of second-order Emden-Fowler neutral delay dynamic equations on time scales. Math. Comput. Modelling, 51, 1221-1229.

Chen, D. -X. \& Liu, J. -C. (2010). Oscillation theorems for second-order nonlinear neutral dynamic equations with distributed delay on time scales. J. Systems Sci. Math. Sci., 30, 1191-1205.

Grace, S. R., Agarwal, R. P., Kaymakçalan, B. \& Sae-Jie, W. (2008). Oscillation criteria for second-order nonlinear dynamic equations. Can. Appl. Math. Q., 16, 59-76.

Grace, S. R., Agarwal, R. P., Kaymakçalan, B. \& Sae-Jie, W. (2009). On the oscillation of certain second order nonlinear dynamic equations. Math. Comput. Modelling, 50, 273-286.

Hassan, T. S. (2008). Oscillation criteria for half-linear dynamic equations on time scales. J. Math. Anal. Appl., $345,176-185$.

Hilger, S. (1990). Analysis on measure chains-a unified approach to continuous and discrete calculus. Results Math., 18, 18-56.

Karpuz, B. (2009). Asymptotic behaviour of bounded solutions of a class of higher-order neutral dynamic equations. Appl. Math. Comput., 215, 2174-2183.

Saker, S. H. (2005). Oscillation criteria of second-order half-linear dynamic equations on time scales. J. Comput. Appl. Math., 177, 375-387.

Saker, S. H. \& O'Regan, D. (2011). New oscillation criteria for second-order neutral functional dynamic equations via the generalized Riccati substitution. Commun. Nonlinear Sci. Numer. Simul., 16, 423-434.

Tripathy, A. K. (2009). Some oscillation results for second order nonlinear dynamic equations of neutral type. Nonlinear Anal., 71, 1727-1735.

Xu, Y. \& Xu, Z. (2009). Oscillation criteria for two-dimensional dynamic systems on time scales. $J$ Comput. Appl. Math., 225, 9-19. 